\title{
Guest editorial: Special issue on Extreme learning machine and applications (I)
}

\author{
Zhihong Man ${ }^{1}$ Guang-Bin Huang ${ }^{2}$
}

Published online: 29 October 2015

(C) The Natural Computing Applications Forum 2015

In the recent development of neural network-based learning, extreme learning machine (ELM) has been attracting a great deal of attention from researchers in different fields. The mechanism of ELM for training single hidden layer feed-forward neural networks (SLFNs) is twofold: First, the input weights of an SLFN are uniformly randomly assigned in a range; second, the output weights of the SLFN are globally optimized, by using the batch learning type of least squares, with a set of training data pairs selected to sufficiently and globally represent the inputand-output data dynamics. Because ELM has the advantages of the fast learning speed, simple implementation and strong robustness against disturbances, it has been widely used for pattern classification, data analysis and system modeling in science, engineering, technology and finance and so on.

In conventional pattern classifications, the dimension of the feature space is often lower than the one of the input pattern space in order to extract the principle features of input data pattern vectors. However, when an SLFN is trained with ELM for a nonlinearly separable pattern classification, the dimension of the feature space (or the number of hidden nodes in the SLFN) is often higher than the one of the input pattern space. The motivation of mapping the input pattern vectors to such a high-dimensional feature space is that all of feature vector clusters are expected to be nondensely distributed in the high-dimensional feature space and then be linearly separated in large extent. The idea of uniformly randomly assigning input

Zhihong Man

zman@swin.edu.au

Swinburne University of Technology, Melbourne, Australia

2 Nanyang Technological University, Singapore, Singapore weights in a range for SLFNs is to realize such a linear separability of feature vectors in the high-dimensional feature space.

The main focus of this special issue is on the recent advances of ELM theory and applications, and the challenges in designing and developing algorithms and systems for science, engineering and industrial applications. In "Extreme learning machine for interval neural networks," the authors use ELM to learn the interval-valued input-and-output dynamics with uncertainties and show the excellent approximation performance compared with the NNs trained with BP. In "Electricity price classification using extreme learning machines," the authors design an SLFN model trained with ELM to classify and predict electricity prices in the deregulated power market. The simulation results with the data of both the Ontario the PJM day-ahead markets have shown the classification accuracy of the proposed SLFN model with ELM. In "Freshwater algal bloom prediction by extreme learning machine in Macau Storage Reservoirs," the authors propose the ELM-based prediction model for phytoplankton abundance in Macau Storage Reservoir. It has been shown in the simulation section that the SLFN model trained with about 8 years of historical data can provide excellent prediction performance compared with the most recent data, and such a research is meaningful for monitoring algal bloom in drinking water storage reservoir. In "Local coupled extreme learning machine," the author assigns an address to each hidden node in the input space, and if or not this hidden node is activated depends on the measure of the similarity between the input pattern and the associated address. It has been shown that the proposed LCELM algorithm can significantly reduce dimensionality of output weights for classifications and regressions in practice. 
In "An efficient query processing optimization based on ELM in the cloud," the authors propose an efficient query processing optimization approach based on ELM in ComMapReduce framework. It has been shown in the experimental section that the scheme can efficiently identify the query processing applications under different communication strategies. In "Principal pixel analysis and SVM for automatic image segmentation," the authors present an automatic object segmentation approach based on principal pixel analysis (PPA) and support vector machine (SVM). The method comprises salient region extraction, principal pixel analysis, SVM training and segmentation. Experiment results on a public benchmark dataset have demonstrated that the proposed method can effectively segment the whole salient object with reasonable better performance and faster speed. In "Applying a new localized generalization error model to design neural networks trained with extreme learning machine," the authors first use the principle component analysis (PCA) to reduce the dimension of the feature space and then design an improved pattern classifier using an SLFN trained with ELM for pattern classification purpose. The results have demonstrated a significant performance improvement of the proposed classifier compared with the existing ones. In "Empirical analysis: stock market prediction via extreme learning machine," the authors develop a neural model based on ELM for predicting stock price movements. It has been shown that the ELM-based model is able to provide fast and accurate predictions compared with many other existing ones.

In "Model predictive engine air-ratio control using online sequential extreme learning machine," the authors develop an online sequential extreme learning machine (OEMPC) for air-ratio regulation of engines. The simulation results have shown that the proposed OEMPC is able to efficiently regulate the air-ratio of engines and the performance is better than the ones using conventional proportional-integral-derivative (PID) controller and the recurrent neural network-based controller. The developed algorithm has been implemented for the air-ratio regulation of a practical engine with good performance. In "Absent extreme learning machine algorithm with application to packed executable identification," the authors propose a novel algorithm based on ELM to process the data with missing features. Considering the fact that both the quadratic function and constraints are convex, the convex optimization techniques can be used to optimally design the output weights of the SLFN to ensure good classification performance. In "MR-ELM: a MapReduce-based framework for large-scale ELM training in big data era," the authors present a novel neural structure with a group of sub-models. After the sub-model is trained with distributed data blocks, they are combined together to form a global system model. The experimental results have shown that such a model is most suitable for the classification and regression of the distributed big data.

In "Orthogonal incremental extreme learning machine for regression and multiclass classification," the author first studies the incremental ELM in detail and then proposes an improved orthogonal incremental ELM, to avoid redundant nodes and thus further reduce the complexity of the SLFN structure. The algorithm has been used for the identification of nonlinear dynamic systems in the simulation section to confirm its efficiency and effectiveness. In "Fast detection of impact location using kernel extreme learning machine," the authors apply kernel ELM to train the SLFN model to predict the impact locations on an aircraft, and the simulation results have firmly shown that the prediction with the proposed algorithm is very accurate compared with a few existing prediction algorithms. It is expected that the results can be used for aircraft condition monitoring and maintenance shortly. In "A new facial expression recognition based on curvelet transform and online sequential extreme learning machine initialized with spherical clustering," the authors present a novel algorithm for facial expression recognition by integrating curvelet transform and online sequential extreme learning machine. The experimental results have shown that the proposed ELM-based classifier has the shortest parameter selection time, highest recognition performance and small testing time.

In "Multispectral palmprint recognition using multiclass projection extreme learning machine and digital shearlet transform," the authors propose a novel multispectral palmprint recognition method based on the MPELM. It has been shown that the method needs less computational time and is capable of obtaining better accuracy than the existing ELM- and SVM-based methods. Experimental results have further demonstrated effectiveness and robustness of the proposed algorithm. In "Improving ELMbased microarray data classification by diversified sequence features selection," the authors present a diversified sequence feature selection-based ELM and the experimental results have shown that the proposed approach significantly improves the efficiency and the effectiveness of ELM against a few existing widely used feature selection techniques.

Finally, we thank all the authors for their great contributions to this special issue. We also thank Neural Computing and Applications and the Editor-in-Chief, Professor John MacIntyre, for the opportunity of publishing these works. 